Pacific Journal of Mathematics

PINCERED BALLS IN MANIFO 


\section{ON PUNCTURED BALLS IN MANIFOLDS}

\section{WolfGANG HeIL}

E. Brown showed that for any map $f$ of a punctured disc $B_{n}$ with $n$ holes into a 2-manifold $M$ that is an embedding of $\partial B_{n}$, there is an embedding $g$ of a punctured disk $B_{k}$ into $M$ such that $g\left(\partial B_{k}\right)$ is a subcollection of $f\left(\partial B_{n}\right)$. In this paper E. Brown's approach is extended to show that a similar result holds for maps of punctured $q$-balls into certain $q$-manifolds $(q \geqq 3)$.

Let $P C(q)$ denote the collection of (topological) $q$-manifolds $M^{q}$ with the property that if $h$ is an embedding of $S^{q-1} \times[0,1]$ into $M^{q}$ that is null homotopic, then $h\left(S^{q-1} \times \frac{1}{2}\right)$ bounds a topological $q$-cell in $M^{q}$.

Note that $P C(1)$ and $P C(2)$ consist of all 1-manifolds and 2manifolds, respectively. It is well-known that $P C(3)$ consists of all 3-manifolds provided the Poincaré conjecture is true in dimension 3 . Since the generalized Poincaré conjecture holds for dimensions $\geqq 5$, [2] we are led to conjecture that $P C(q)$ consists of all (topological) $q$-manifolds for $q \geqq 5$, particularly since, from the proposition below, if $h: S^{q-1} \rightarrow \partial M^{q}$ is an embedding such that $h\left(S^{q-1}\right)$ is null-homotopic in $M^{q}$, then $M^{q}$ is indeed a $q$-cell $(q \geqq 5)$. However, C. McA. Gordon, whom I would like to thank most sincerely for providing the proof of the following proposition, informs me that C. T. C. Wall and John Morgan have counter examples for $q>4$.

Proposition. Let $C \cong S^{q-1}$ be a boundary component of a compact $q$-manifold $M$. If $[C]=0$ in $\pi_{q-1}(M)$, then $M$ is contractible.

Proof. Let $q \geqq 3$. By the Whitehead and Hurewicz Theorems it suffices to show that $\pi_{1}(M)=1$ and $H_{*}(M)=0$. Now $\partial M=C$ since otherwise $[C] \neq 0$ in $H_{q-1}(M)$. Also, $M$ is orientable since otherwise for the orientation cover $M^{\prime}$ of $M$ we have $\partial M^{\prime}=C^{\prime} \cup C^{\prime \prime}$ (copies over $C$ ) and $\left[C^{\prime}\right]=0$ in $\pi_{q-1}\left(M^{\prime}\right)$, a contradiction.

There is a map $f:\left(B^{q}, S^{q-1}\right) \rightarrow(M, \partial M)$ such that $f \mid S^{q-1}$ is a homeomorphism. Orient $M$ so that $f$ has degree 1 . Then for the fundamental classes $z_{q}, w_{q}$ in $H_{q}\left(B^{q}, S^{q-1}\right), H_{q}(M, \partial M)$, resp., we have $f^{*}\left(z_{q}\right)=w_{q}$ and a commutative diagram

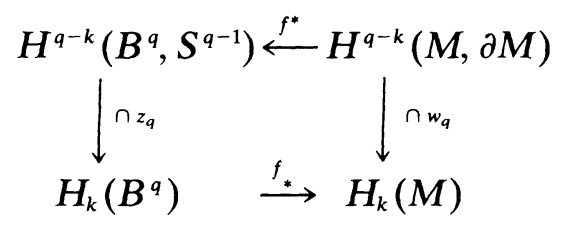


By Lefschetz duality, the vertical maps are isomorphisms. Therefore $f_{*}\left(-\cap z_{q}\right) f^{*}$ is an isomorphism. It follows that $f_{*}$ is onto and hence that $H_{*}(M)=0$.

To show that $\pi_{1}(M)=1$, let $p: \tilde{M} \rightarrow M$ be the universal covering. Then $f$ lifts to $\tilde{f}:\left(B^{q}, S^{q-1}\right) \rightarrow(\tilde{M}, \partial \tilde{M})$. But $1=\operatorname{deg}(f)=$ $\operatorname{deg}(p \circ \tilde{f})=(\operatorname{deg} p)(\operatorname{deg} \tilde{f})$, hence $\operatorname{deg} p= \pm 1$ and $\pi_{1}(M)=1$.

For $q \geqq 2, n \geqq 1$, let $B_{n}^{q}$ be a punctured $q$-ball with $n-1$ holes, i.e., $B_{n}^{q}$ is obtained from $S^{q}$ by removing the interiors of $n$ mutually disjoint $q$-balls.

For a bicollared $S^{q-1} \subset M^{q}$ let $N \approx S^{q-1} \times I$ be a neighborhood of $S^{q-1}$ and let $M^{\prime}=\operatorname{cl}(M-N) \cup B^{\prime} \cup B^{\prime \prime}$, where the boundaries of the $q$-balls $B^{\prime}, B^{\prime \prime}$ are attached to the boundary components $S^{q-1} \times 0$ and $S^{q-1} \times 1$ of $\operatorname{cl}(M-N)$. We say $M^{\prime}$ is obtained from $M$ by surgery along $S^{q-1}$. Let $X$ be the space obtained from $M^{\prime}$ by identifying $B^{\prime}$ and $B^{\prime \prime}$ under a homeomorphism. Note that $X$ can be obtained from $M^{q}$ by attaching a $q$-ball $B$ to $S^{q-1}$ along its boundary and $X-B=$ $M^{\prime}-\left(B^{\prime} \cup B^{\prime \prime}\right)=M-S^{q-1}$.

Lemma. Let $S$ be a $(q-1)$-sphere in $X-B$. If $S \simeq 0$ in $X$, then $S \simeq 0$ in $M^{\prime}$.

Proof. Suppose $S^{q-1}$ separates $M$ into $M_{1}$ and $M_{2}$; then $M^{\prime}=$ $M_{1}^{\prime} \cup M_{2}^{\prime}$, where $M_{1}^{\prime}=M_{1} \cup B^{\prime}, M_{2}^{\prime}=M_{2} \cup B^{\prime \prime}$. Let $X_{1}^{\prime}$ be obtained from $M_{t}$ by collapsing $S^{q-1}$ to a point. The projection $p: X \rightarrow X_{1}^{\prime} \vee X_{2}^{\prime}$ is a homotopy equivalence which sends $S$ into $X_{1}^{\prime}$, say. This can be seen as follows: Identify a neighborhood of $S^{q-1}$ with $N=S^{q-1} \times[-1,1]$, where $S^{q-1}=S^{q-1} \times\{0\}$. Let $w$ be the "centerpoint" of $B$ and for $y \in S^{q-1}$ let $r(y)$ be the "radius" in $B$ from $y$ to $w$. In $X_{1}^{\prime} \vee X_{2}^{\prime}$ we identify $p(N)=\left(S^{q-1} \times I\right) /\left(S^{q-1} \times\{0\}\right)$ with the cones over $S^{q-1} \times\{-1\}$ and $S^{q-1} \times$ $\{1\}$ wedged together at their vertices to a vertex $v$. Let $g: X_{1}^{\prime} \vee X_{2}^{\prime} \rightarrow X$ be the map that is the identity outside $p(N)$ and which sends the join of $x$ and $v$ (for $x \in S^{q-1} \times\{-1\}$, respectively $S^{q-1} \times\{1\}$ ) linearly to $x \times$ $[-1,0] \cup r(x \times\{0\})$, resp. $x \times[0,1] \cup r(x \times 0)$. Then it is clear that $g$ is a homotopy inverse of $p$. But since $X_{1}^{\prime}$ is a retract of $X_{1}^{\prime} \vee X_{2}^{\prime}$ it follows that $S \simeq 0$ in $X_{1}^{\prime}$ already and hence in $M_{1}^{\prime} \simeq X_{1}^{\prime}$.

If $S^{q-1}$ does not separate $M$, let $\tilde{X} \rightarrow X$ be the infinite cyclic covering of $X$ determined by $B$ : the $q$-ball $B$ lifts to $q$-balls $\cdots B_{-1}, B_{0}, B_{1}, \cdots$ and each component of $\tilde{X}-\bigcup_{i=-\infty}^{\infty} B_{i}$ maps homeomorphically onto $X-B$. For each $i$, let $X_{\iota}^{\prime}$ be obtained from $M^{\prime}$ by collapsing $B^{\prime}$ and $B^{\prime \prime}$ to single points. There is a projection $\tilde{X} \rightarrow \vee_{i=-\infty}^{\infty} X_{1}^{\prime}$ that is a homotopy equivalence and hence $\pi_{q-1}\left(X_{j}^{\prime}\right)$ injects into $\pi_{q-1}(\tilde{X})$, for each $j$. Let $\tilde{S}$ be a lift of $S$ to $\tilde{X}$. Then $\tilde{S}$ lies in a component of $\tilde{X}-\cup B_{i}$ and is mapped into a factor $X_{j}^{\prime}$ of $\vee X_{i}^{\prime}$. It follows that $\tilde{S} \simeq 0$ in $X_{j}^{\prime}$, hence $S \simeq 0$ in $M^{\prime}$. 
THEOREM. Let $f: B_{n}^{q} \rightarrow M^{q}$ be a map such that $f \mid \partial B_{n}^{q}$ is a bicollared embedding, $f\left(\partial B_{n}^{q}\right)=S_{1} \cup \cdots \cup S_{n}$. Suppose that the manifold $M^{\prime} o b$ tained from $M^{q}$ by surgery along $S_{i}(i=2, \cdots, n)$ belongs to $P C(q)$. Then some subcollection of $\left\{S_{1}, \cdots S_{n}\right\}$ contains $S_{1}$ and bounds an embedded punctured $q$-ball in $M$.

Proof. By Brown's result we can assume that $q \geqq 3$. Let $X$ be obtained from $M$ by attaching $q$-balls $B_{i}$ to $S_{i}(i=2, \cdots, n)$ along their boundaries. Then $X-\bigcup_{i=2}^{n} B_{l}=M^{\prime}-\bigcup_{i=2}^{n} B_{i}^{\prime} \cup \bigcup_{i=2}^{n} B_{i}^{\prime \prime}$, where $B_{i}^{\prime}, B_{i}^{\prime \prime}$ are the balls used for surgery on $S_{i}$. Now $S_{1} \approx 0$ in $X$. By the lemma, $S_{1} \simeq 0$ in $M^{\prime}$. Since $M^{\prime} \in P C(q), S_{1}$ bounds a $q$-ball $B_{*}$ in $M^{\prime}$. Let $E$ be the component of $B_{*}-\bigcup_{\imath=2}^{n}\left(B_{\imath}^{\prime} \cup B_{\imath}^{\prime \prime}\right)$ which has $S_{1}$ on its boundary. If for each $i=2, \cdots, n$ only one of $\partial B_{i}^{\prime}, \partial B_{i}^{\prime \prime} \subset \partial E$, then $E$ is the desired punctured ball in $M$ bounded by $S_{1}$ and some of the $S_{i}$ 's. In fact, this is the only case that can happen. For suppose for some $i, \partial B_{i}^{\prime}$ and $\partial B_{i}^{\prime \prime} \subset \partial E$. Then let $k$ be a simple arc in $E$ from a point of $\partial B_{i}^{\prime}$ to a point on $\partial B^{\prime \prime}$, such that $k$ misses the other $\partial B_{j}$ 's and such that $k$ corresponds to a simple closed curve in $M$ that intersects $S_{1}$ in one point

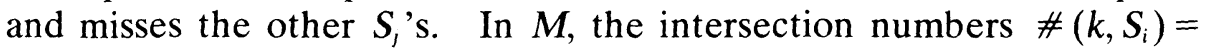
\pm 1 , but $\#\left(k, \Sigma_{l \neq l} S_{l}\right)=0$, which is impossible since $S_{i} \sim \bigcup_{j \neq i} S_{l}$.

\section{REFERENCES}

1. E. M. Brown, A note on punctured disks in a 2-manifold, Proc. Amer. Math. Soc., 22 (1969), 471.

2. M. H. A. Newman, The engulfing theorem for topological manifolds, Ann. of Math., 84 (1966), 555-571.

Received February 6, 1976 and in revised form July 28, 1976.

Florida State University

TAllahassee, FL 32306 




\section{Pacific Journal of Mathematics}

Vol. 68, No. 1

March, 1977

Richard Julian Bagby, On $L^{p}, L^{q}$ multipliers of Fourier transforms . .......

Robert Beauwens and Jean-Jacques Van Binnebeek, Convergence theorems in

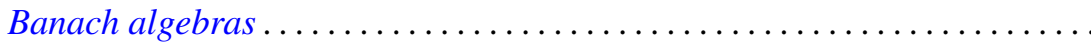

James Cyril Becker, Skew linear vector fields on spheres in the stable

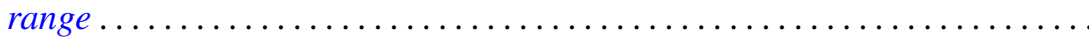

Michael James Beeson, Continuity and comprehension in intuitionistic formal

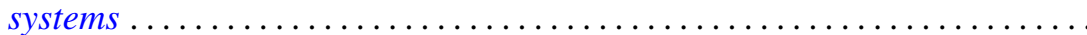

James K. Deveney, Generalized primitive elements for transcendental field

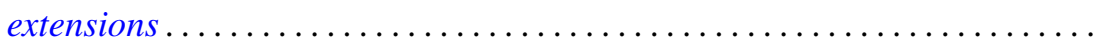

Samuel S. Feder, Samuel Carlos Gitler and K. Y. Lam, Composition properties

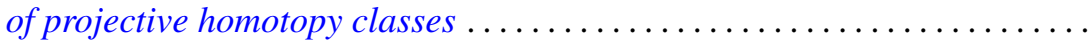

Nathan Jacob Fine, Tensor products of function rings under composition ......

Benno Fuchssteiner, Iterations and fixpoints . . . . . . . . . . . . . .

Wolfgang H. Heil, On punctured balls in manifolds

Shigeru Itoh, A random fixed point theorem for a multivalued contraction

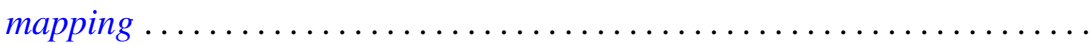

Nicolas P. Jewell, Continuity of module and higher derivations . . . . . . ......

Roger Dale Konyndyk, Residually central wreath products . . . . . . . . . . .

Linda M. Lesniak and John A. Roberts, On Ramsey theory and graphical

parameters.

Vo Thanh Liem, Some cellular subsets of the spheres.

Dieter Lutz, A perturbation theorem for spectral operators

P. H. Maserick, Moments of measures on convex bodies ... . . .

Stephen Joseph McAdam, Unmixed 2-dimensional local domains . .

D. B. McAlister and Norman R. Reilly, E-unitary covers for inverse semigroups...

William H. Meeks, III and Julie Patrusky, Representing codimension-one

homology classes by embedded submanifolds . . .

Premalata Mohapatro, Generalised quasi-Nörlund summability . .

Takahiko Nakazi, Superalgebras of weak-*Dirichlet algebras .

Catherine Louise Olsen, Norms of compact perturbations of operators .

William Henry Ruckle, Absolutely divergent series and isomorphism of

subspaces. II.

Bernard Russo, On the Hausdorff-Young theorem for integral operators .

Arthur Argyle Sagle and J. R. Schumi, Anti-commutative algebras and

homogeneous spaces with multiplications ............

Robert Evert Stong, Stiefel-Whitney classes of manifolds .

D. Suryanarayana, On a theorem of Apostol concerning Möbius functions of

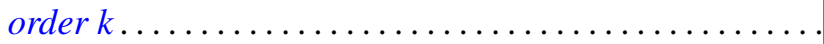

Yoshio Tanaka, On closedness of $C$ - and $C^{*}$-embeddings . . 